\title{
Retention of root canal posts in vitro
}

\author{
Retention of prefabricated and individually cast root canal posts in vitro

\section{S-0. Hedlund, N. G. Johansson and G. Sjögren Br Dent J 2003; 195: 155-158}

\section{Objectives}

The aim of the study was to evaluate the retention of prefabricated root canal posts made of a variety of materials that have recently been introduced into dentistry.

\section{Materials and methods}

The posts studied were CosmoPost, Composipost carbon fibres, Composipost Æstheti-Plus, Composipost Light-Post and Para Post Fiber White. The posts were luted in extracted human premolars and the cores were built up with the resin composites recommended by the manufacturers. The retention of individually cast gold alloy posts luted with zinc-phosphate cement were used as reference. A universal testing machine was used to determine the retention of each cemented post. Data were compared using ANOVA supplemented with Fisher's PLSD at a significance level of $\mathrm{p}<0.05$

\section{Results}

Only the CosmoPost system exhibited retention values that were significantly lower than for the conventionally cast gold alloy posts luted with zinc-phosphate cement. The force necessary to loosen the CosmoPost specimens was significantly less than that needed to loosen the Composipost Æstheti-Plus $(p<0.05)$ and the Composipost Light-Post systems ( $p<0.001)$. The force necessary to loosen the Para Post Fiber White specimens was significantly less than for the Composipost Light-Post system $(p<0.01)$. Other combinations did not differ significantly $(\mathrm{p}>0.05)$.

\section{Conclusions}

When zirconium oxide ceramic posts are luted with resin composites the bonding between the ceramic and resin composite seems to be weak. Further studies are thus necessary to achieve improved retention of all-ceramic root canal posts.

\section{IN BRIEF}

- There have been concerns expressed about metal root canal posts and cores with regard to corrosion, risk of root fracture and aesthetic outcome. Therefore, alternative non-metallic posts and cores have been introduced into dentistry.

- This study examines the retention of some prefabricated root canal posts made of a variety of materials that have been recently introduced into dentistry.

- This study gives information about the retention in vitro of prefabricated root canal posts made of a variety of materials. To assess their suitability for use as an alternative to individually cast posts controlled clinical studies are indicated.

\section{COMMENT}

Posts are an essential component of the armamentarium involved with the retention of crowns and bridges. A 'standard' post is cast gold with a zinc phosphate cement. Failure of the post, usually by loosening, will lead to catastrophic failure of the crown / bridge. Concerns about possible toxic effects of the metals used in posts and the adverse stress concentrations due to the mismatch in physical properties between the post, lute and tooth have lead to the development of ceramic and composite posts using a variety of filler reinforcements such as carbon and quartz fibres.

In this ex vivo study the force required to loosen ten samples of five of these newer posts luted into extracted human premolars was measured and compared with standard cast gold. As with all studies of this type the 'clinical force' necessary for a satisfactory system is unknown, but under the assumption that the gold is satisfactory, forces less than this can be considered to be clinically unsatisfactory. The statistical analysis can be criticised as there are no power calculations.

It was found that CosmoPost, a zirconium dioxide ceramic system, had a statistically significant lower loosening force than cast gold, $89 \mathrm{~N}$ compared with $179 \mathrm{~N}$. The highest was Composipost Light-Post with $261 \mathrm{~N}$.

The results had considerable scatter, reflecting the variations inherent in the premolars. It was observed that the predominant locus of failure of the CosmoPost system was adhesive at the lute ceramic interface. This is an important observation as it reflects the problems in achieving a satisfactory bond to a ceramic surface, and this may limit the clinical usefulness of this system unless pretreatment such as sandblasting is carried out.

Martyn Sherriff, Senior Lecturer in Dental Biomaterials Science, GKT Dental Institute doi:10.1038/sj.bdj.4810402 